\title{
Nocturnidad y Noctis: Consideraciones para la etnografía de trabajo de producción de nocturnidad
}

\author{
Nocturnidad \& Noctis: Considerations for the nighttime work ethnography
}

\author{
Julio César Becerra Pozos'
}

\begin{abstract}
Resumo
Partiendo de la reflexión teórica que procura hacer una distinción de la noche como algo más que un ciclo natural, considerándola una configuración socio espacial y temporal, cargada por la subjetividad de todos los que participan en ella, así como de estructuras culturales y relacionales; articulándola desde lo laboral, se propone analizarla desde dos facciones sumamente interconectadas: noctis y nocturnidad. Centrando la propuesta de este artículo en la nocturnidad; mediante el estudio etnográfico de las características siu generis del trabajo de producción de interacciones realizado por meseros y meseras en espacios semi privados de venta y consumo de alcohol en dos subconjuntos de bares de la Ciudad de México, se conceptualiza a dicha actividad como un trabajo no clásico de producción de nocturnidad. Más adelante, se presentan las consideraciones y adaptaciones a las técnicas y estrategias de investigación utilizadas, además de los desafíos éticos y prácticos que se generan en las etnografías de trabajo de nocturnidad. Finalmente, se hace hincapié en la necesidad de una conceptualización configuracional y ad hoc para el estudio etnográfico de las multiplicidades del trabajo de economía nocturna en el servicio de producción de interacciones.
\end{abstract}

Palavras chave: nocturnidad; trabajo no clásico; performance laboral; economía de tiempo nocturno; etnografía

\begin{abstract}
Starting on a theoretical reflection, in which the night is a social, spacial temporal configuration, full of the subjectivity of the cultural and relational structures of those who participate on it, rather than just a natural cycle; once joint with the labor perspective, it's the proposal of this article to analyze the night from it's two highly related components: noctis and nocturnidad. The considerations and adaptations to the research technics and strategies used, along with the ethical and practical challenges from the ethnography of the nocturnity work, comes next. Finally, the emphasis on the need of an ad hoc configurational conceptualization in the ethnographic study of nighttime economy work diversity.
\end{abstract}

Keywords: ethnography; nocturnity; night labour; non classic work; night time economy.

\footnotetext{
1 Doutorando em Estudios Sociales na Universidad Autónoma Metropolitana, Iztapalapa, México. Email: juliocesarbecerrapozos@gmail.com.
} 


\section{Introduciendo el trabajo realizado en bares $^{2}$ y a la nocturnidad}

Desde su concepción, nos enfrentamos al desafío de la caracterización del trabajo de meseros de bares y que hemos ubicado como un Trabajo no clásico y emocional (DE LA GARZA, 2011, 2011b, 2017; HOSCHILD, 1987, 2016; WHARTON, 2009), estético y sexualizado (WARHUST, 2009; SANDIFORD, 2010, 2012) dentro del rubro de los servicios de la economía de tiempo nocturno (NTE') (GRAZIAN, 2007, 2009; CHATTERTON, 2002, 2004; SU-JAN, 2014; MATEO \& DÍAZ, 2009).

Característico de este trabajo también son los distintos requerimientos a partir de la condición de género, alta volatilidad del mercado laboral, precarias condiciones de trabajo, (para la gran mayoria) falta de prestaciones, importancia de la sociabilidad en la permanencia y acceso al empleo, así como un predominante carácter etario juvenil en el que la naturaleza de la construcción del ingreso es siempre inestable, pues aunque se trata de trabajadores asalariados, su principal constituyente es la propina.

Hay veces que namás sales con veinte pesos, que con cincuenta, o si sacaste cien dices, pues ya, al menos ya estuvo lo del dia (Osiel, 23 años. Mesero, casado y con un hijo. Ciudad de México 2016)

Pero uno vive bien porque cuando uno es mesero, cuanto te llevas por día como mil quinientos, eso es arriba de cualquier profesional; te puedes llevar hasta dos mil pesos de propina en un buen lugar [bar], por día, arriba de dos mil pesos, ósea jodidamente en un buen lugar, tipico de la condesa donde, donde llegan los politicos ¿no? te llevas dos mil pesos de propina, mínimo ochocientos y te estás quedando con todos (I: ajá) no nada más eres tú, son seis meseros por turno cuatro meseros y estas con todos como, ahi no mames, nos fue de la chingada ochocientos varos, unos llegamos a los mil (Bere, 33 años. Mesera de alto capital cultural. Ciudad de México 2017)

Sin embargo, se distinguen de otros trabajos de interacción directa (cara a cara) con la clientela en el sentido de que no solamente tienen su

\footnotetext{
2 Cuando se utiliza el término bares en cursivas se refiere en modo abstracto y conceptual a los espacios semiprivados, asociados a la producción de nocturnidad, de venta y consumo (de al menos) alcohol y en los que la generación del servicio está mediada por la interacción cara a cara entre trabajadores clientes y representante del capital (C-T-K); de manera generalizada, se engloban bares, cantinas, antros, clubes, discotecas (si es que aún existen), salones, micro bares, table dances, cafebrerias, karaoke bar, pub, irish pub, english pub, lounge, gastrobar, tasca, mezcalería, cerveceria, cerveceria artesanal, club, jazz club, bohemian club, rock club, classic bar, art bar, galerias bar, o cualquier otro nombre que resulte afin a las características ya enunciadas.

3 También llamada Night Time Economy por sus siglas en inglés.
} 
mayor demanda física y simbólica durante la noche, sino que es un trabajo asociado a la producción de la nocturnidad y en el que las interacciones pueden gestarse en estados alterados de conciencia (EAC), general, aunque no exclusivamente, inducidos por alcohol tanto en clientes, trabajadores y representante(s) del capital.

A la diferenciación simbólica y laboralizada de la noche entre noctis y nocturnidad se refiere el presente trabajo; viéndolo como una propuesta para la práctica etnográfica de este ámbito laboral, sustentamos la pertinencia del uso conceptual de la nocturnidad entendida como una compleja construcción social-espacial (TALBOT, 2007; SU JANG, 2014; CHATTERTON, 2002, 2004; LINDÓN, 2012; BENNET, 1997; MATEO \& DÍAZ, 2009; CUNNINGHAM, 1980, PARKS, 2003; AGUIRRE, 2000; ESQUER, 2006; AGOFF, 2012; MARGULIS, 1995; GRAZIAN, 2007, 2009), una configuración social única e irrepetible para cada uno de los que participan en ella, que si bien ocurre durante el tiempo nocturno natural (la cual llamaremos simplemente, noche), resulta de una demanda lúdica específica asociada al consumo, con un carácter evidentemente urbano y cuya espacialidad no se extiende a toda la ciudad sino a específicos circuitos que poseen liminalidades difusas.

En ese sentido, cómo bien mencionan Chatterton (2002), Farrer (2004) y Talbot (2009); en el espacio nocturno convergen diversas clases, (media, baja, alta) y nacionalidades -quizá con un predominio de la clase media y popular- en la que satisfacen sus demandas de interacción social y cultural además de esa búsqueda de divertimento nocturno que devenga en una experiencia particular, subjetiva y auténtica en cuanto a su unicidad.

Además, es aquí donde a partir de la noción básica de que la noche en su sentido conceptual es un constructo social, o en palabras de Margulis "la noche es una categoria socialmente construida, determinada por procesos históricos y culturales" (1994: 15), es posible incorporar la temporalidad y el espacio al considerar que el pasaje del día a la noche opera en la naturaleza significativa del espacio, por lo que, aunque pudiera parecer una obviedad, la noche solo existe a un tiempo en latitudes geográficas 
específicas mientras que la nocturnidad ocurre en tiempos y espacios específicos e interconstruídos.

Por tanto, sustentamos la relevancia de consideraciones teóricas de espacio con el tema de investigación en tres premisas:

La primera refiere a que lo social no sucede en el vacío, por lo que no se debe ignorar la construcción e injerencia del espacio tanto este, como en cualquier otro tema de investigación. La necesidad de dar muestra de las razones de la diversidad y complejidad que existe en el trabajo de producción de la nocturnidad e interacción directa con el cliente tanto en los trabajadores, como en los lugares de venta y consumo, así como en quienes los frecuentan es la segunda premisa.

Por tanto, las variantes y coincidencias observadas tanto en la operación del establecimiento laboral como en la experiencia subjetiva de los trabajadores (meseros y meseras) en su interrelación con clientes y representantes del capital puede ser explicada a partir de la perspectiva del espacio concebido y vivido, lo cual "constituye una forma de habitar parcialmente en el sentir de otro cuerpo. Por otra parte, la intercorporalidad también da cuenta de la posibilidad de ser parte de las experiencias sensoriales de otros" (LINDÓN, 2013, p.6).

En tercer lugar; el carácter de nocturnidad inviste a la actividad laboral y a la tríada ya antes mencionada (Trabajadores-ClientesRepresentante del capital), por tal, esta no debe ser tomada como un mero contexto temporal de oposición al día, sino que el ciclo nocturno en este rubro tiene una relevancia específica en la cotidianeidad de quienes lo viven y significan por lo que la nocturnidad en su noción compleja y relacional se convierte en uno de los ejes articuladores para el análisis realizado por trabajadores de bares.

El trabajo de esta índole procura un servicio que denote unicidad y personalización, pero para ello se debe reparar en las tendencias que genera la interrelación del espacio y atmósfera en sí (bar), la clientela, y el trabajador con todas las combinaciones que ello implica. Al final de este artículo se presenta el uso de la distinción entre trabajo nocturno o por turnos (noctis) y trabajo de producción de nocturnidad (nocturnidad); este 
último como articulador de la subjetividad del trabajo generada entre los miembros de la tríada cliente-capital-trabajo a la par que da importancia a la construcción de la atmósfera de nocturnidad en su sentido amplio, y que simultáneamente, permite teorizar sobre los conflictos laborales que conlleva una actividad de este tipo, es decir: un performance laboral para la nocturnidad.

\section{Noche y Trabajo / Nocturnidad y Noctis}

A partir de este punto, nos referiremos por el uso simple de Noche, a la noción natural del periodo durante el que una parte de la tierra, por acción de la rotación, deja de recibir la luz solar y, por ende, permanece en oscuridad (IZQUIERDO, 1992); es decir, una noción natural y no necesariamente social. Empero, la Nocturnidad será toda aquella significación social, cultural, temporal y espacial que se configura de manera compleja con quienes participan de ella, la noche en su sentido lúdico, transgresor, normativo y permisivo. Naturalmente, la noche llega al término de cada día, pero la efervescencia de la nocturnidad se manifiesta con mayor dominio durante los fines de semana.

Claro está que la oferta de ocio nocturno solo parece agotarse o al menos reducirse significativamente durante el lunes y parte del domingo y aunque es común desde los martes encontrar lugares de venta y consumo de alcohol con algunos clientes, el cenit de la producción de nocturnidad indudablemente ocurre en viernes y sábado; dicho de otra manera, aunque desde el inicio de semana hay funcionamiento de bares, la concurrencia no suele ser tan elevada salvo algunos cuyo reconocimiento público en los circuitos de nocturnidad sea especialmente como un día en específico (ejemplo, los miércoles de música en vivo en el Club Atlántico de la Ciudad de México, o para un caso más evidente, los lunes de Severino en Buenos Aires).

Por ende, la mayor afluencia de clientes y el interés de las observaciones a estos espacios laborales de nocturnidad ocurre en las noches del viernes y el sábado, cualidad que se exacerba si se trata del fin de semana inmediato al pago general de salarios (quincena o inicio de mes), en 
ese sentido, en cuanto a la relación de la Nocturnidad con la Noche, podemos decir que mucho de su sentido de espacialidad es la oposición a lo diurno, el espacio que se hace diferente en los significados que las prácticas poseen.

El trabajo nocturno (Noctis) tiene relación con el de nocturnidad en el sentido que genera discontinuidades con el ciclo circadiano de los trabajadores, irrumpe en la cotidianidad, tareas de reproducción y sociabilidad con familiares y amistades, por tanto, es coincidente con algunos aspectos del segundo y considerando que este estudio se centró en los procesos y subjetividad laborales, aunque relacionado, abordarlo enteramente desde la noción natural de noche forzaria analíticamente un estudio etnográfico de trabajadores de nocturnidad.

Otra diferenciación que podemos situar entre Noctis y Nocturnidad es que la segunda considera al espacio diurno como el propio de los prolegómenos del trabajo y del mantenimiento, el tiempo de las tareas de reproducción. Aunque la nocturnidad de los clientes será sustancialmente diferente a la de quienes la laboran (meseros, bartenders, personal de seguridad, hostess, etc.) es mediante la función e interés del capital, que ambas se conjugan.

Por otro lado, si el énfasis de la Nocturnidad está dado por las prácticas nocturnas relacionadas al ocio, lo permisivo y lúdico, también venimos de una definición no explicitada en que la noche es mucho más que juego y diversión; mientras que la Noctis es para la gran mayoría descanso, reposo y dominio de lo privado y la intimidad.

La excepcionalidad que se construye socialmente todas las noches (menos lunes) en nocturnidad, a diferencia de las prácticas hegemónicas (extendidas socialmente), son prácticas disruptivas del orden de la noche y día a nivel biológico. Se vuelven un conjunto de prácticas que lindan en las fronteras de la permisividad y eso lo permite la condición liminal que implica la Nocturnidad: negociar los límites de lo socialmente establecido.

Finalmente, de la vertiente del espacio concebido vivido el cual tiene un particular enfoque en la subjetividad de los sujetos y su experiencia cotidiana es que a partir de las teorizaciones que devienen de la time geography; everyday rythms; proxémica, diastémica así como nearby 
territories, se puede dar una profundidad mayor al análisis de las significaciones y percepciones emocionales en los lugares de ocio nocturno (bares).

Dentro de ese tiempo delimitado como nocturno la noche en la ciudad se vuelve depositaria de lo lúdico, lo sensual, el erotismo y la vía urbana de la recreación (simulación) de la fiesta. De este modo, la nocturnidad significa iluminar lo oscuro; visibilizar lo que no estaba descubierto y la noche es más el espacio de lo privado, la intimidad, el descanso y las tareas de reproducción. Esto es, se construyen lugares de nocturnidad que son distintos a otros espacios diurnos. La noche es tiempo de reposo con imaginario hegemónico de lo que es la noche, la nocturnidad es lo contrario, es inventar la ciudad de la noche. Para entender la ya mencionada distinción del carácter social y simbólico de la noche, así como su relación con la construcción de nocturnidad y trabajo, se incluye el siguiente esquema.

Cabe aclarar, reconocemos que el día también es configurado por una vasta complejidad simbólica, cultural, estructural y social por lo que, reducirlo a una generalidad como "Día" no refleja la utilidad que un concepto amplio de espacio y tiempo significaría para el estudio de la esfera de lo laboral en el trabajo diurno. 


\section{Esquema de distinción laboral de la noche}

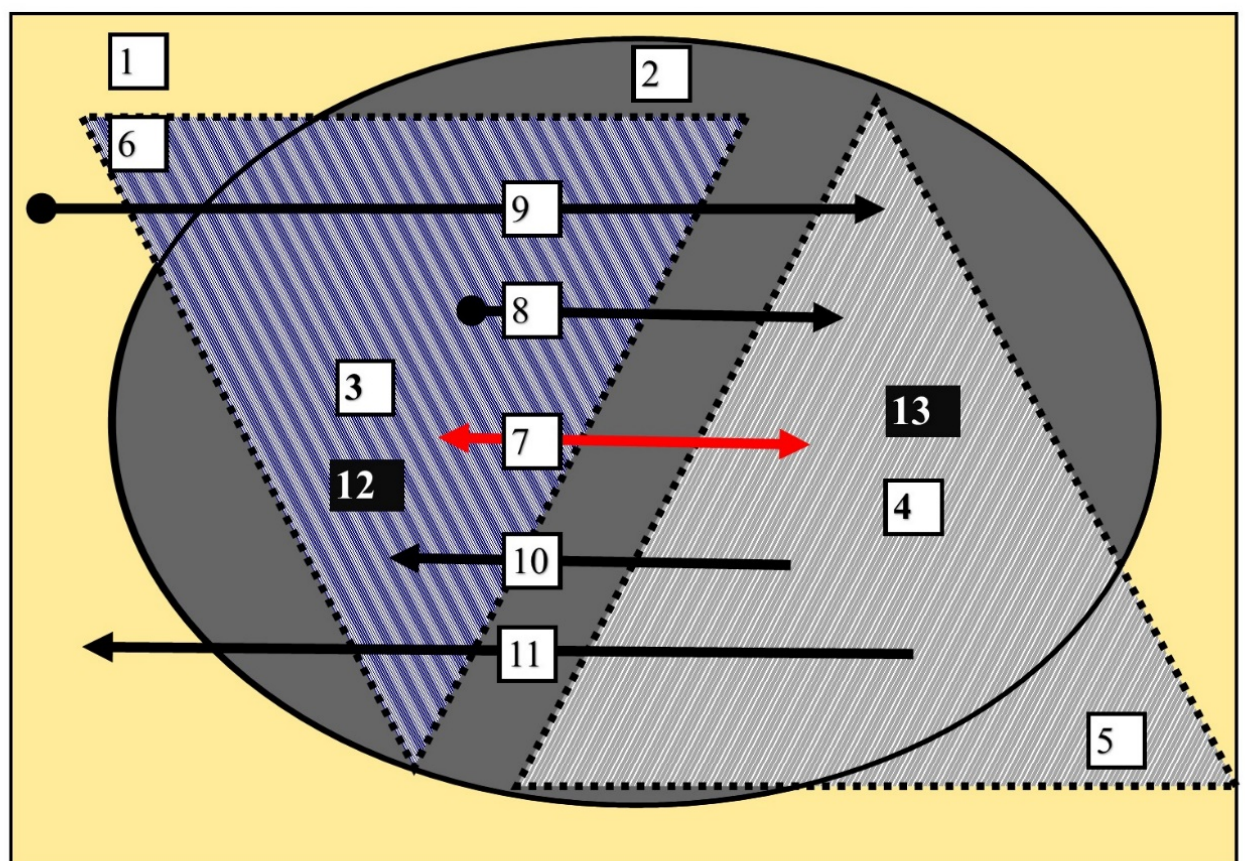

Fig. 1 Esquema de distinción laboral de la noche. Elaboración propia. Noviembre 2017

1. Día (en sentido natural de ciclo de rotación)

2. Noche (en sentido natural del ciclo de rotación)

3. Noctis

4. Nocturnidad

5. Construcción de Noctis que puede suceder durante el día

6. Construcción de Nocturnidad que puede suceder durante el día

7. Espacios y sujetos laborales con movilidad continua entre Noctis y Nocturnidad

8. Acción de transición de la Noctis a la Nocturnidad en trabajadores

9. Transición Día-[Noche]Noctis-Nocturnidad en trabajadores

10. Transición [Noche] Nocturnidad a Noctis en trabajadores

11. Transición [Noche] Nocturnidad-Noctis-Día en trabajadores

12. Trabajadores nocturnos

13. Trabajadores de producción de nocturnidad

A continuación, la descripción de cada uno de los puntos:

I y 2) Día y noche en sentido natural de ciclo de rotación. 
Como se indica en el nombre, es una mera alusión al movimiento de rotación y traslación que genera el ciclo natural de lo que conocemos como día y noche; se trata de una diferenciación sin carácter simbólico o cultural, pero si visual y con inferencia en los ciclos circadianos de los sujetos laborales.

\section{3) Noctis}

La noctis, de ninguna manera, es la parte residual de la nocturnidad, sino un complemento por el cual algunos sujetos laborales transitan de una a otra dentro de una misma noche. Noctis, es la parte de la noche en su sentido más clásico, la referida a las tareas de reproducción de la fuerza de trabajo y el descanso, pero dentro de la concepción de espacio como socialmente construido e irrepetible; en una configuración multidimensional, dinámica y sensitiva que va más allá de la substancia medible o tangible (LINDÓN, 2006, 2007 y 2013).

Se "duerme de noche y se vive de dia" versa una canción del grupo Calle 13; empero, la noctis no queda exenta de trabajo, es aquí donde podrían ubicarse los turnos nocturnos del mundo fabril, las guardias de los hospitales, el trabajo de los veladores y así. Es decir, aunque predominantemente está configurada por el descanso, la intimidad y el espacio privado (el hogar); ello no significa que carezca de actividad laboral y social en diversos espacios dentro de la misma noche. Esta distinción de la noche no tiene necesariamente un carácter rural o urbano, pero no existe de manera natural, sino que es una construcción social.

Asimismo, con las limitantes y controversias que el concepto sugiere, podría decirse que la noctis es, en sentido amplio, la parte cotidiana de la noche y complementaria, en campo biológico y psicosocial, del mundo del trabajo diurno.

\section{4) Nocturnidad}

La nocturnidad, entendida como una compleja construcción socialespacial, una configuración social única e irrepetible para cada uno de los que participan en ella. 
Aunque predominantemente ocurre durante el tiempo nocturno natural (el cual llamaremos Noche en sentido natural del ciclo de rotación), resulta de una demanda lúdica específica asociada a un consumo particular, configuración cargada de contradicciones y con un carácter evidentemente urbano, cuya espacialidad no se extiende a toda la ciudad sino a específicos circuitos que poseen liminalidades difusas. Al igual que la noctis, no existe una sola manera de experienciar la nocturnidad, sino que está atravesada por la subjetividad, deseo y expectativa de cada uno de los que participan en ella y que puede ocurrir más allá de la noche.

Va acompañada de una lógica comercial de consumo recreativo asociado al alcohol, la sexualidad y la permisividad pero al mismo tiempo, es una arena de contradicciones pues en varios espacios también tiene un carácter sumamente restrictivo, excluyente y normativo ${ }^{4}$; por tanto, la nocturnidad no necesariamente debe ser siempre transgresora, disruptiva ni juvenil, pero sí acompañada de una economía de tiempo nocturno [Night time economy] (TALBOT, 2007; SU-JAN, 2014) e iniciar, terminar o atravesar la Noche.

5) Construcción de Noctis que puede suceder durante el día

En el esquema se muestra como una esquina del triángulo Noctis, pero fuera del óvalo de Noche puesto que hace alusión a que la noctis puede ser recreada fuera del ciclo de la noche, pensándolo desde los trabajadores nocturnos y quienes laboran en la producción de la nocturnidad; se trataría de una transición de la noctis al Día como el replicar las condiciones y prácticas para el descanso e intimidad/privacidad fuera de la noche. Ejemplo, cuando un médico concluye su guardia nocturna y al llegar a casa realiza su rutina de descanso, generando un ambiente de poca luz, sin intromisiones y silencio para entregarse al descanso y lo que hay antes de ello, "cenar", ponerse el pijama, cepillarse los dientes.

\footnotetext{
${ }^{4}$ Por ejemplo, códigos de vestimenta bien delimitados, que no es algo exclusivo de bares de élite pues en algunos "alternativos" pueden ser excluyentes para outsiders.
} 
6) Construcción de Nocturnidad que puede suceder durante el día

La posición en el esquema de este punto hace referencia a que la Noche y el Día no son el factor único en la Nocturnidad pues esta, al ser un constructo social, también puede darse durante el Día mediante dos modos de reproducir la nocturnidad durante el día. Uno es el que refiere a la reconstrucción de la noche en espacios privados y completamente cerrados que procuran aislar al consumidor de su noción de temporalidad; casinos, que generalmente operan 24 horas al día, siete días a la semana; que en su interior no existen relojes, ventanas ni ningún otro indicio del exterior.

Igualmente sucede en los bares de table dance que, aunque no suelen operar en una intensidad tan fuerte como los casinos, también funcionan durante el día y procuran extender la noche en su interior, careciendo de ventanas y con una cuidada iluminación tenue y neón.

El segundo modo, no se centra en el espacio en sí, sino en ejercer prácticas de nocturnidad durante el dia, ya sea al inicio o al final de este, pero suele terminar, iniciar o atravesarse a la temporalidad de la noche. Es decir, sucede cuando las practicas descritas en el punto de nocturnidad se llevan a cabo en un tiempo diurno, ejemplo de ello pudiese ser el caso brasileño en el cual la jornada de nocturnidad (lo que incluye la apertura de bares y su concurrencia) inicia en horarios en los que aún es de día, igualmente sucede en fines de semana coincidentes con el pago de quincena en que es común ver como los establecimientos y posiciones laborales propios de la economía de tiempo nocturno ya están habilitados y con fuerte demanda desde el horario de "after office" que inicia alrededor de las seis de la tarde, generando una liminalidad difusa y choque con el mundo laboral, social y diurno de otros.

También valdría la pena mencionar como en algunos casos espacios públicos como malecones, parques y calles de barrio tienen un halo de permisividad que permite extender la jornada de nocturnidad al tiempo diurno cuando esta no concluyó durante la noche, a ello podrían sumársele los bares de after que operan hasta cerca del mediodia. 
7) Espacios y sujetos laborales con movilidad continua entre Noctis y Nocturnidad

El carácter movible y mutable de la noctis con la nocturnidad se hace evidente mediante un análisis de estos sujetos y espacios laborales pues en ellos se expresa la posibilidad de transición entre una y otra construcción. Al recordar que la noctis no carece de actividad laboral y que la nocturnidad requiere del trabajo de muchos para su producción; resulta natural pensar que ambas configuraciones tienen puntos de encuentro y de transición ya que coinciden en el espacio geográfico de la ciudad. Los sujetos laborales que más comúnmente pueden ser identificados cómo transicionales entre noctis, nocturnidad y viceversa son los conductores de transporte personal privado como taxistas, radiotaxis, Uber y aplicaciones afines, pero en su jornada nocturna.

Por cuestiones de tiempo y alcance, no es posible ahondar sobre ellos desde una perspectiva teórica, sin embargo, sí resultan ejemplares para el punto anterior descrito; aunque sus traslados no son exclusivamente de transición de noctis a nocturnidad y viceversa, llevar pacientes a un hospital, realizar encomiendas, movilizar a trabajadores nocturnos (veladores, trabajadores de fábrica en turno nocturno, médicos, etc.) y semejantes suceden dentro de la misma noctis. Así como trasladar clientes de un bar a otro no significa salir de la construcción de nocturnidad; en este esquema de trabajo en la nocturnidad y noctis cómo ponemos énfasis en su característica laboral de transición entre una y otra.

Durante la jornada nocturna suelen transportar a potenciales clientes que, desde su noctis o trabajo diurno se dirigen a una particular experiencia deseable de la nocturnidad, pero su jornada también se extiende a la vuelta, también trasladan a trabajadores, clientes trasnochados, jóvenes (y adultos) en estados alterados de conciencia, parejas románticas y demás variedades en cuanto a grupos etarios, culturales y de diversa índole socioeconómica de vuelta a la noctis 5 .

\footnotetext{
5 Otro ejemplo de trabajo transitorio entre las dos configuraciones ya mencionadas es el de operador de minisúper, kiosko, depósito o "tiendita" de 24 horas pues, aunque estos se encuentran en un espacio bien delimitado e inamovible durante su jornada nocturna proveen insumos interactúan con sujetos en su noctis y en nocturnidad
} 
Para el caso de los primeros, la nocturnidad entra en contacto con ellos no únicamente por la interacción con sujetos participantes, y a los cuales transportan (es decir, en el interior del auto), sino también se requiere un mayor estado de alerta pues circulan por el mismo espacio público con otros vehículos cuyos conductores pueden encontrarse en algún estado alterado de conciencia lo cual genera un mayor peligro y les obliga a desarrollar habilidades particulares para su jornada nocturna en contacto con la nocturnidad.

8 y 9) Acción de transición de la Noctis a la Nocturnidad en trabajadores; Transición Día[Noche]Noctis-Nocturnidad en trabajadores

Aunque es válido el debate de si para el trabajador de producción de nocturnidad su jornada obedece a la noctis en lugar de la nocturnidad, a nuestro parecer, el carácter impredecible y los particulares requerimientos fisicos, emocionales, estéticos y performáticos de su actividad, cómo se verá más adelante, hacen que los ubiquemos una figura laboral siu generis de productores de nocturnidad. Dentro de esa lógica, aunque su horario de trabajo suele iniciar durante el día a primeras horas de la noche, esta primera parte de la jornada cuyo trabajo es más de actividades de limpieza y preparación con un bajo flujo de clientela, la flecha de direccionalidad hace referencia a como dentro de una misma jornada, y en un mismo espacio, se puede transitar de la noctis a la nocturnidad en la actividad laboral sin que ello signifique una movilidad física geográfica.

I0 y II) Transición [Noche] Nocturnidad a Noctis en trabajadores; Transición [Noche] Nocturnidad-Noctis-Día en trabajadores

A diferencia de los dos puntos anteriores, aquí si implica una transición física pues que se trata de una movilidad asociada al término de la jornada laboral de producción de nocturnidad. Nos parece prudente incluirla pues durante el trabajo de campo resultó una constante dentro de los inconvenientes del trabajo realizado en bares, en especial en los que se dan en zonas de evidente gentrificación, las problemáticas para el regreso al hogar procurando no genere un gasto extra. En tal sentido, las opciones más comunes suelen ser compartir un taxi o Uber entre varios de los compañeros 
de trabajo o tener un acuerdo ya preestablecido con un trabajador del volante con una mejor tarifa. ${ }^{6}$

\section{2) Trabajadores nocturnos}

Dentro de la configuración de noctis podrian enlistarse trabajos exclusivamente nocturnos como el de velador; así como complementarios al día o que tienen un equivalente diurno como pudiese ser el de operador de fábrica en turno nocturno, guardia médica, servicio de urgencias, empleado de mostrador de 24 horas, restaurantes de cadenas en 24 horas, así como trabajadores de limpia y otros servicios públicos pero se trata de actividades que se realizan durante la noche por la des intensificación de sus flujos productivos. ${ }^{7}$

\section{3) Trabajadores de producción de nocturnidad}

Nos remitimos a enlistar como figuras laborales arquetípicas de la nocturnidad a bartenders, bailarinas exóticas, chippendales, meseros(as) de bares, prostitutos(as), dealers, DJ's, personal de seguridad, cadeneros, showmans, hosts, hostess, y músicos por contrato (profesionales y amateurs, de todo tipo de género musical e instrumentos, solistas o en agrupación) e itinerantes, siempre y cuando laboren en espacios dedicados a la producción de la nocturnidad entre otros que pudiesen sumarse a esta lista.

Una vez que se ha hecho la diferenciación entre noctis y nocturnidad; a nuestro parecer, en los estudios laborales actuales es necesaria una diferenciación conceptual para quienes trabajan durante la noche y aquellos que laboran para producir una configuración particular de la noche. Aunque existe un concepto bien consolidado de Trabajo nocturno o trabajo por turnos, el cual ha sido profundizado desde el enfoque psicosocial y biológico;

\footnotetext{
6 Para aquellos que viven y trabajan en las cercanías de la ruta del trolebús (único transporte público de 24 horas) se vuelve la mejor opción, otros prefieren esperar a que comience el horario de servicio de otras opciones como el Metrobús, aunque dependiendo del conocimiento del trabajador acerca de las opciones de movilidad que ofrece la CDMX, usualmente el retorno al hogar implica un viaje largo y a menudo tiempos de no trabajo y espera que terminan por alargar la temporalidad de la jornada laboral.

7 Permiten tareas de mantenimiento y suplementarias (supermercados y algunas fábricas); o que la intensidad del trabajo es continua o necesaria las 24 horas del dia (aeropuertos, hospitales, farmacias, personal de hoteleria) además de aquellas que requieren de una pasividad que la intensidad diurna no da cabida (trabajadores de limpia pública, obras públicas y afines) entre otras variedades que han escapado a nuestra breve consideración.
} 
el trabajo de producción de nocturnidad continúa siendo visto desde aristas que no necesariamente son reflejo de su configuración. Algunos estudios lo sitúan desde el enfoque de trabajo sexualizado, trabajo de los servicios, o trabajo de producción de interacciones; enfoques válidos pero que de verse en conjunto y añadiendo las características particulares de la nocturnidad ayudarian a un mejor entendimiento de la problemática laboral por la que atraviesan estos actores.

\section{Performance laboral de meseros de bares de la CDMX}

Una vez definida la Nocturnidad para el trabajo realizado en bares, ahora es posible ahondar sobre la importante injerencia que ocupa el uso de performance laboral en la configuración del trabajo de producción de nocturnidad; este concepto se retoma en tres pasos: El primero correspondió a la revisión y selección de diversas posturas que han teorizado sobre dicho concepto, el segundo se enfoca en visibilizar la existencia de un performance asociado a la nocturnidad, en sentido general y sin hacer alusión a la práctica laboral; en tercer lugar se encuentra la laboralización del concepto de performance de nocturnidad; el cual pone en juego, junto a otros elementos de carácter subjetivos, emocionales, estéticos, etarios, genéricos y fenotípicos, así como un saber hacer aprendido en la experiencia; el conjunto de la particular configuración de los meseros y meseras trabajadores(as) en bares.

En consideración que el performance genera "la participación plena en una relación social, indica la realización corpórea (y muchas veces artística) de una obra, de un texto, o hasta de una idea (...) [y] que une relaciones sociales y semióticas." (JOHNSON, 2014, p.12) Además de tener un carácter perlocucionario en el cual es imposible saber el resultado de los actos performáticos en interacción con otros actores (AUSTIN, [1955] 2001) pero que a su vez sí tiene una intencionalidad definida (GOFFMAN,[1956] 2001; BELL, 1976) puesto que prevalece una concientización de la actuación, así, es posible reflexionar sobre la pertinencia de hablar de un rizoma de performatividades de nocturnidad. 
Rizoma en el sentido de que no existe un solo modo de performance de nocturnidad, sino que la multiplicidad de este será tan variada como la subjetividad de cada uno de los sujetos que participen en ella, sin embargo, consideramos es posible marcar algunas tendencias.

Para articular lo anterior con la nocturnidad tendriamos qué, participar en la experiencia de la misma, lo que implica una inmersión de interrelaciones sociales y procesos de significación asociados con lo lúdico, lo transgresor y lo permisivo o bien con lo normativo, pautado y controlado pero que depende de la expectativa y deseo de cada uno de los participantes; es decir, los sujetos que coproducen la nocturnidad no necesariamente actúan del mismo modo que en su cotidianeidad diurna.

La nocturnidad abre la posibilidad de identificarse con actitudes o personificaciones de otros grupos etarios (ya sea en la propia trayectoria o a modo de emulación, asimismo, hacia lo joven o lo adulto); asenso o descenso en la escala social (gente de altos estratos socioeconómicos que por aventura, se "disfrazan" y atienden a sórdidos espacios alternativos o de contracultura así como jóvenes de clase trabajadora $u$ oficio que el fin de semana -si el fenotipo se los permite- acuden a bares de élite) o incluso movilidad en preferencias y construcciones de género puesto que la nocturnidad, al menos en las grandes metrópolis, es efervescente, efimera y receptáculo del anonimato. Incluso el acto de salir con amigos (actitudes propias de la construcción de masculinidades, obligación de alcanzar o fingir un estado anímico o de ebriedad, etc.) o en pareja (no evidenciar agotamiento o cansancio, exponer diferentes capitales sociales, culturales, literarios, musicales, etc.) a un lugar ad hoc a la posición y preferencia social de pertenencia no quedan exentos de un particular actuar o deber ser asociado a la nocturnidad. Con ello no afirmamos que la nocturnidad sea enteramente una fachada o falseamiento de individuos, con los ejemplos anteriores nos referimos a en qué momentos en particular se puede hablar de un performance de nocturnidad.

La expectativa, prenociones e imaginarios que atraviesan a los diversos performances de nocturnidad guardan una estrecha relación con los modos de ejercer el trabajo de producción de nocturnidad, para este caso, el 
de meseros y meseras de bares. A raíz del interés por conocer la configuración del trabajo de producción de nocturnidad e interacciones realizado por meseros en dos subconjuntos de bares; se ha ido dibujando la relevancia del performance laboral, el communitas y la subjetividad en la propina como elementos que podrian enriquecer y articular la dicha configuración entre trabajador(es)[meseros], cliente(s) y el representante(s) del capital.

Por tal razón, para el estudio del trabajo en bares centrado en los meseros (as), se procura dar un uso del concepto de performance 8 que articule la subjetividad del trabajo generada entre los miembros de la triada cliente-capital-trabajo; que dé importancia a la construcción de la atmósfera de nocturnidad en su sentido amplio y que destaque aquello que resulta sui generis de este trabajo a la par que permita teorizar sobre los conflictos laborales que conlleva una actividad de este tipo, es decir: un performance laboral asociado a la nocturnidad que nos permite resaltar dos configuraciones de producir el servicio, una en la que el trabajo procura evidenciarse, y otra, en la que se disimula en una supuesta horizontalidad. Esta distinción no es menor pues, en el grueso de la producción teórica europea y norteamericana del tema, no se realiza esta diferenciación a menos que sea mediante una visión de estudios de género.

Es decir, pareciera que el trabajo de esta índole fuera homologado en cuanto a que se procura un servicio que denote unicidad y personalización, pero sin reparar en las tendencias que genera la interrelación del espacio y atmósfera en sí (bar), la clientela, y el trabajador con todas las combinaciones que ello implica. Consideramos que el trabajo de interacción cara a cara realizado en el conjunto de estudio tiene fuertes dispositivos performáticos; a razón de la fuerte carga simbólica que contiene el trabajo, el velo de nocturnidad y el hecho de que las calificaciones y requerimientos del trabajador de esta particular actividad no pueden ser homologados a los del trabajo fabril; sustentamos lo anterior en cuanto que su actividad laboral

\footnotetext{
8 Se optó por mantener el anglicismo de performance sin una traducción a performatividad o semejantes para evitar confusiones en las distintas connotaciones que se le atribuyen a lo largo del texto. Es por ello por lo que se ha marcado en cursivas a excepción de las citas, en ellas se ha respetado la traducción del autor.
} 
requiere de capacidades subjetivas, afectivas y corpóreas que son aprendidas y perfeccionadas a través de la adquisición (consciente o no) de un saber hacer obtenido mediante la experiencia laboral y cuya evaluaciónsignificación del servicio se refleja no en el salario, sino en el ingreso mediante la propina.

Reiterando que el bar no sólo oferta alcohol sino que también se asiste por diversión, por distracción, interacción-sociabilidad y una atractiva posibilidad de salir de rol cotidiano que se traduce como potencialidad; una potencialidad que se extiende al trabajador que puede llegar a convencer al cliente de que es su amigo, que le agrada o que está enteramente a su servicio de igual manera que tiene la posibilidad de invertir la relación de subordinación y hacerle creer que por el contrario, él le está concediendo un favor al cliente al brindarle un servicio. ${ }^{9}$

Entre la liminalidad y permisividad potenciada por la nocturnidad, existe la posibilidad de que clientes, trabajadores e incluso el representante del capital adopten consciente o inconscientemente actitudes e identidades que pueden ser consideradas como una suerte de performance que es interconstruído por la atmósfera de nocturnidad, el espacio nocturno y los miembros de la tríada ya mencionada.

De vuelta a la relación del uso del concepto performance con el trabajo realizado en bares, la propuesta de Anne W. Jhonson (2014) la cual refiere a la noción de un rizoma de asociaciones de actos palabras relacionados con él, así como la doble hélice semántica del concepto la cual se refiere a que el performance genera la participación completa en una relación social (JOHNSON, 2014).

Por otro lado, indica la realización corpórea (y muchas veces artística) de una obra, de un texto, o hasta de una idea [...] pero la fuerza de la palabra convertida en concepto es precisamente la unión de los dos sentidos: la doble hélice semántica del performance, indeterminadamente ondulatoria y particular, que une relaciones sociales y semióticas (JOHNSON, 2014, p.12) .

Esta definición se ha considerado como óptima para dar cuenta de la diversificación y alcance del concepto. También se añaden complementos

\footnotetext{
${ }^{9}$ Este acto sucede generalmente en bares de carácter exclusivo, aunque quizá donde es más evidente esta tendencia en el rol simbólico que desempeñan los "cadeneros"
} 
como el de los actos perlocucionarios de Austin ([1955] 2001) referentes a la imposibilidad de anticipar el resultado de un performance. La importancia de la construcción social del género (tanto de masculinidades como feminidades) y la posible desigualdad de poder vistas desde los estudios de género y feminismo de Sedgwick (2003), Butler (1997) y Pasko (2002) no resulta ajena a este estudio en que la sexualidad y el género juegan un papel importante entre las interacciones cliente-capital-trabajo. Del mismo modo que la construcción espacial de estos lugares también es decisiva en los diversos modos de performance que ahí se gestan, recordamos que lo espacial no refiere a una mera construcción física ni de elementos estéticos tangibles sino también en su función subjetiva, dinámica, interconstruída y no representacional.

Casi para finalizar, consideramos que el performance no debe ser visto desde una atomización del concepto, ya que para poder hablar de una performatividad asociada a la nocturnidad, con carácter laboral y enfocada a un fin instrumental de ingreso expresado en la propina, se debe articular con teoria de espacio, identidades, significación en torno a la propina, los nuevos estudios laborales (principalmente con la teoría del trabajo no clásico) e incluso estudios de mercado y etnográficos, pensar en performance resulta otra vía para sintetizar la manera en que se ponen en juego diversos significados, emociones y capitales; incorporados y atravesados por características fenotípicas, estéticas, etarias, genéricas y sexualizadas.

Esas características, en la interacción con el cliente, configuran una actividad laboral que a su vez pudiese ser reducida a una mera funcionalidad mecánica de proveedor de insumos (también hay meseros que llevan la interacción al mínimo e incluso evitan el contacto aunque ello no signifique que no haya una performatividad en el acto). De tal manera que, aunque puede ser considerado como un trabajado evidentemente simbólico y estético, al mismo tiempo, en su función básica, podría llevarse a cabo sin ellas.

Hasta cierto punto en la empresa era lo que se manejaba era, $<<$ si tú estás mal pues dejas eso afuera y aquí vienes y te pones con una sonrisa>> de cierta manera si está un poco pesado estar con eso porque hay dias en los que te sientes mal y no quieres estar de <<ay 
estoy feliz>> pero por el concepto del bar sí tienes que estar así (Jessica 23 años. Mesera y estudiante. Ciudad de México. 2017)

Como que también te acostumbras al tipo de clientes que visitan, ósea te aburres hasta el cierto punto de ese tipo de gente, te aturdes y si causa un tipo de desesperación aunque creo, y ahí es algo personal, como que todo está en decir, no pus aguante, relájate, tu chamba está chida y tienes que hacer como que fiesteas que si no pues no sale (Laura 26 años con cinco de experiencia como mesera. Ciudad de México, 2016)

\section{Consideraciones finales a la etnografía de trabajo nocturno}

Además de la necesaria diferenciación analítica entre noctis y nocturnidad para la práctica etnográfica del trabajo de producción de nocturnidad; lo que se evidenció como unidad fundamental fue la subjetividad laboral regida por un actuar particular que debe adecuarse a la construcción espacial y temporal donde se da la práctica del trabajo. Por tanto, consideramos que uno de los principales resultados logrados fue el de aportar, en alguna medida, sobre la producción y vivencia de la nocturnidad entendida como una construcción socio espacial estrechamente relacionada al mundo del trabajo.

Este apartado realizado con la información construida del análisis del trabajo de campo y que refiere a lo concerniente a los diversos modos y efectos en que la interacción de los meseros de bares también destaca que pueden estar mediadas por estados alterados de consciencia (EAC) por uno o más de los actores junto a los que los meseros realizan su trabajo.

La disrupción en la interacción es generada por la clientela; generalmente referente a estados de ebriedad con todas sus implicaciones, así como las estrategias que el trabajador debe desarrollar para mediar con dichos estados a la par de mantener abierta la posibilidad de un buen término de la interacción que tiene como objetivo (para el trabajador) la obtención de la ganancia por medio de la propina.

Ya cuando los ves hasta la madre sabes que ya valió, una de dos, o te vas a rayar o te regresas a casita con las manos vacias (...) también luego les pega la depresión y la soledad y te quieren comprar, bueno no, más bien te agradecen que los hayas escuchado, que les des consejo y asi; y ya hasta te agradecen de verdad y te dan una buena propina (Martín, mesero, 29 años. Ciudad de México, 2016) 
De ahí que las reuniones, generalmente asociadas a un consumo etílico, al término de la jornada laboral o al menos una vez por semana; cumplen una función práctica e instrumental para el representante del capital, ya que de ese modo refuerza vínculos afectivos y se generan procesos de reciprocidades obligadas (GRANOVETTER, 1985).

Entre los trabajadores persiste la noción de que la propina es algo que se vuelve reflejo del trabajo verdaderamente realizado, mientras que el salario no. El resultado del trabajo realizado de manera individual se refleja en la propina, mientras que el salario es indiferente a la productividad del trabajador, pensándolo desde la performatividad laboral; la propina tiene una valencia tanto instrumental como simbólica, en la que el punto cenit en la interacción cliente-trabajador es al momento de la dádiva o pago de la misma; además de ser el momento en el que se puede dar por concluida la relación en la producción del servicio.

Vale la pena mencionar qué, en la gran mayoria de las entrevistas, principalmente en los meseros hombres, era común que los informantes incluyeran una anécdota referente a algún cliente que les dejó un monto inusualmente alto como muestra de empatía o gratitud por su servicio. Se trata de experiencias que marcaron su trayectoria laboral y que en alguna medida les permiten legitimarse como hábiles para la actividad.

Esta actividad laboral tiene elementos ambiguos, contradictorios y complejos, que obligan a tomar con cautela cualquier juicio generalizado y dificultan el encontrar cifras confiables respecto a la magnitud de la ocupación. Es un trabajo caracterizado por la precariedad laboral en seguridad social, prestaciones y estabilidad, pero que a la par, dependiendo de diversos capitales del trabajador, puede proveer altos ingresos económicos por medio de la propina.

Por tanto, al tener en cuenta lo anteriormente descrito; del intento de caracterizar al trabajo realizado por meseros de bares, tenemos como resultado que es un Trabajo no clásico de producción simbólica y Relación laboral ampliada (DE LA GARZA, 2011, 2011b); que además está dentro del rubro de los servicios (KORCZYNSKI, 2002, 2005; BROOK, 2007; SALAMAN, 2007; HERNÁNDEZ, 2011) y cuidados (MOLINIER y ARANGO, 2011) y 
fuertemente asociado al trabajo emocional (SEYMOUR, 2005; HOSCHILD, 1987, 2016; WARHUST, 2009).

Con una fuerte valoralización y subjetividad que se le atribuye a la propina (SOSTERIC,1996; GRAZIAN,2007,2009; RAINE, 2012), cuyo ingreso suele ser superior al del salario por lo que requiere de un saber hacer aprendido en la experiencia que despliega elementos de un performance particular (NIETO,2016; JOHNSON,2014; GOFFMAN,[1956] 2001; BELL,1976; LINDÓN,2013; ALEXANDER,1988, 2005; SEDGWICK,2003).

Performatividad atravesada por la nocturnidad y en la que las construcciones de género, tanto de feminidades (GUADARRAMA, 2007; NASH, 2006; VARGAS, 2003; WARHUST2009; SANDIFORD; 2010,2012; ESQUER Y AGOFF, 2012) como masculinidades (GUTTMAN,1997; JÍMENEZ, 2007; MOSSE,1996; BRANDES, 1991 Y SEDGWICK:1995) intervienen, junto con los elementos anteriormente descritos, en la configuración del trabajo de meseros de bares.

En este tipo de lugares, al menos los que son asi como el XXXXXX, sabes que los dones que vienen a echarse sus chelas también te van a estar sabroseando pero de ahí no va a pesar si no te dejas, ósea, se vale que te vean así como que, pues acá feo o sabroseándote, pero mientras tu no des pauta a más, no va a pasar nada, también saben que aqui está Rodrigo (personal de seguridad) en cuanto alguno se quiere pasar yo si armo un panchote que hasta se disculpan y no lo vuelven a hacer o ni vuelven al bar, es eso, no me quita nada que me miren, y si no eres simpática ni te dejan propina, pero tampoco es un bar de putas (Rocío, 24 años. Mesera/artista. Ciudad de México, 2017)

Por último, aprovechamos la oportunidad para recalcar que, tras la revisión de trabajos afines a la nocturnidad, resaltamos la poca atención que se ha dado a quienes laboran activamente en su producción y en particular a la nebulosa atención que se ha puesto en los trabajadores que no resultan "protagonistas" de la noche, centrándose en las figuras más representativas o visibles del rubro como stripers, cadeneros, músicos y bartenders pero menos destacables en la plantilla laboral como lo son meseros, seguridad y limpieza, aunque imprescindibles para montar y atender la oferta de nocturnidad cada noche, parecen quedar olvidados por quienes frecuentan y estudian la noche. 
Primero, la fiesta del mesero comienza después de que se le acaba la fiesta a todo mundo, excepto a pocos. Son lugares más extremos, son lugares que sirven cerveza o en algunos casos son lugares que la fiesta comienza a las cinco y termina a las once de la mañana, entonces, estás agarrando el horario que muy pocas personas agarran. Automáticamente la fiesta cambia. Todos se la saben, todos son personas nocturnas; no vas a ver nadie como aqui que se echan tres cervezas y vomitan. Allá, si vomitan, llegan al baño y le atinan porque llevan años vomitando igual (...) son todas las personas que conocen a su cuerpo, que saben que tanto se van a hacer bien o mal, ósea son personas que viven en la noche y por lo tanto se mueven muy bien en la noche. Corres más riesgo de que te roben, te golpeen (...) pero todos nos sabemos los mismos trucos, todos trabajamos en los mismo, entonces, todos venimos con la misma insanidad de sacar toda la tensión, por lo tanto, son fiestas más explosivas, más viscerales ya que estás sacando todas las ganas reprimidas de enfiestarte en el bar porque uno actúa como que está en la fiesta, pero es parte del trabajo (Moka, 27 años. Mesera, personal de seguridad, gerente).

\section{Referências}

AGUIRRE, Jorge. Los usos del espacio nocturno en el puerto de Veracruz. Estudios sobre las Culturas Contemporáneas, vol.6, n.12, 2000.

ALEXANDER, Jeffrey. Pragmática cultural: Un nuevo modelo de performance social. Revista Colombiana de Sociologia, n. 24, p.9-67, 2015.

AUSTIN, J.L. Cómo hacer cosas con palabras [Online]. Escuela de Filosofia, Universidad ARCIS. Disponivel em: http://revistaliterariakatharsis.org/Como_hacer_cosas_con_palabras.pdf. Acesso em: 30/07/2018 [1955].

BELL, Michael. Tending Bar at Brown's: Occupational Role as Artistic Performance. Western Folklore, vol.35, n.2, 1976.

BENNET, Andrew. 'Going down the Pub!': The Pub Rock Scene as a Resource for the consumption of popular music. Popular Music, vol.16, n.1, 1997.

BERICAT, Eduardo. El trabajador de fin de semana en la sociedad del ocio: Tiempo de trabajo y calidad de vida en el sector servicios. Revista Internacional de Sociologia (RIS), v.38, 2004.

BUTLER, Judith. Mecanismos psíquicos del poder: Teorias de la sujeción. Madrid: Ediciones Cátedra, 2001 [1997].

CHATTERTON, Paul; HOLLANDS, Robert. Theorising Urban Playscapes: Producing, Regulating and Consuming youthful Night life city space. Urban Studies, vol.39, n.1, 2002.

DE LA GARZA, Enrique. El Trabajo no clásico y la ampliación de los conceptos de la Sociología del Trabajo. Revista trabajo, vol.8, n.10, 2012. 
n.68, 2013.

Trabajo no clásico y flexibilidad. CADERNOS CRH, Salvador, v.26,

"Hacia un concepto ampliado de trabajo, de control, de regulación y de construcción social de la ocupación: los otros trabajos". In: CORTÉS, E.; DE LA GARZA, E (org.). Teorias sociales y estudios del trabajo. Barcelona: Anthropos, 2008.

“Trabajo no Clásico, organización y Acción Colectiva”. In: DE LA GARZA, E. (org.). Trabajo no Clásico, Organización y Acción Colectiva. Madrid: Plaza y Valdés, vol.2, 2011.

"Trabajo atípico, ¿Identidad o Fragmentación?: alternativas de análisis". In: DE LA GARZA, E. et al (org.). Trabajos atipicos y precarización del empleo. Ciudad del México: El Colegio de México, 2011b.

DI MÉO, Guy. L'Homme, la Société, l'Espace. París: Anthropos, 1991.

DÍAZ Cruz, Rodrigo. La vivencia en circulación. Una introducción a la antropología de la experiencia. Alteridades, vol.7, n.13, 1997.

ESQUER, Fernández; AGOFF, María Carolina. Drinking and working in a cantina: misrecognition and the threat of stigma. Culture, Health \& Sexuality, vol.14, 2012.

FARRER, James. Urban Nightscapes: Youth Cultures, Pleasure Spaces, and Corporate Power. Contemporary Sociology: A Journal of Reviews, vol.33. 2004.

GOFFMAN, Erwing. The presentation of self in everyday life. Edinburg: University of Edinburgh Social Sciences Research Center, 1956.

GRAZIAN, David. On the Make: The Hustle of Urban Nightlife. Chicago: University of Chicago Press, 2008.

Urban Nightlife, Social Capital, and the Public Life of Cities. Sociological Forum, vol.24, n.4, 2009.

GUADARRAMA, Rocío, TORRES, José Luis Torres (org.). Los significados del trabajo femenino en el mundo global: Estereotipos, transacciones y rupturas. Barcelona: Anthropos, 2007.

HOSCHILD, Arlie. The Managed Heart: Commercialization of Human Feeling. California: University of California Press, 1987.

Invisible labour: hidden work in the contemporary world. California: University of California Press, 2016.

JIMÉNEZ Guzmán, María Lucero; GUERRERO, Olivia Tena. Reflexiones sobre masculinidades y empleo. Cuernavaca: Universidad Nacional Autónoma de México, 2007. 
JOHnSON, Anne W.; CRUZ, Rodrigo Díaz. Presentación. Alteridades, vol.24, n. $48,2014$.

JOHNSON, Anne. ¿Qué hay en un nombre?: una apología del performance. Alteridades, vol.24, n.48, 2014.

KORCZYNSKI, Marek; KERFOOT, Deborah. Gender and Service: New Directions for the Study of 'Front-Line' Service Work. Gender, Work and Organization, vol.12, n.5, 2005.

KORCZYNSKI, Marek. Human Resource Management in Service Work. Londres: Palgrave, 2002.

LINDÓN, Alicia. La ciudad y la vida urbana a través de los imaginarios urbanos. Revista Eure, vol.33, n.99, 2007.

Los imaginarios urbanos y el constructivismo geográfico: los hologramas espaciales. Revista Eure, vol.33, n.99, 2007b.

Performatividades urbanas: la construcción social de la ciudad a través de los cuerpos que la habitan. XXIX Congreso Latinoamericano de Sociologia-2013: Crisis y Emergencias Sociales en América Latina, Chile 2013.

"Territorialized everydayness, Between proxemics and diastemics: Space-time rhythms in a context of acceleration". In: PIRANI, Blanca Maria; SMITH, Thomas S. Body and Time: Bodily Rhythms and Social Synchronism in the Digital Media Society. Cambridge: Cambridge Scholars Publishing, 2013b.

LÓPEZ Ojeda, Andrés. "Formas simbólicas y tránsitos identitarios. Una aproximación teórico metodológica a la percepción, uso y apropiación de la noche entre los enfermeros y enfermeras nocturnos". In: GUADARRAMA, Rocío; TORRES, José Luis (org.). Los significados del trabajo femenino en el mundo global: Estereotipos, transacciones y rupturas. Barcelona: Anthropos, p.123-142, 2007.

MARGULIS, Mario et. al. La cultura de la noche: La vida nocturna de los jóvenes en Buenos Aires. Buenos Aires: Biblos, [1994] 2005.

NASH, Merry. Identidades de género, mecanismos de subalternidad y procesos de emancipación femenina. Revista CIDOB d'Afers Internacionals, núm.73-74, 2006.

NIETO, Raúl Calleja. Trabajo en la globalidad hegemónica. Performance laboral en México y Guatemala. Revista Andaluza de Antropologia, n.11, sept. 2016.

NOFRE, Jordi; DIAZ, Mateo. Ocio nocturno, gentrificación y distinción social en el centro histórico de Sarajevo. Anales de Geografia de la Universidad Complutense, vol.29, n.1, 2009. 
PASKO Lisa. Naked Power: The Practice of Stripping as a Confidence Game. Sexualities, v.5, n.1, 2002.

PRED, Allan. Social Reproduction and the Time-Geography of Everyday Life. Geografiska Annaler, Series B, Human Geography, vol.63, n.1, 1981.

SANDIFORD, Peter; SEYMOUR, Diane. Serving and consuming: drink, work and leisure in public houses. Work, Employment \& Society, v.27, n.1, 2013.

SANDIFORD, Peter. The concept of occupational community revisited: analytical and managerial implications in face-to-face service occupations. Work Employment \& Society, vol.21, n.2, 2007.

SEDGWICK, Eve Kosofsky. Touching Feeling: Affect, Pedagogy, Performativity. Durham: Duke University Press, 2003.

SEYMOUR, Diane. Learning emotion rules in service organizations: socialization and training in the UK public-house sector. Work Employment Society, vol.19, n.3, 2005.

SOSTERIC, Mike. Subjectivity and the Labour Process: A Case Study in the Restaurant Industry. Work Employment \& Society, vol.10, n.2, 1996.

SU-JAN, Yeo. An (Extra)ordinary Night Out: Urban Informality, Social Sustainability and the Night-time Economy. Urban Studies, vol.51, n.4, 2014.

TALBOT, Deborah. Regulating the Night: Race, Culture and Exclusion in the Making of the Nighttime Economy. Nova York: Ashgate, 2007.

VARGAS, Lilia Esther. "El otro del género". In: MATALOBOS, Isabel Jaidar (org.). Convergencias en el campo de la subjetividad. Unidad Xochimilco: Universidad Autónoma Metropolitana, 2003, p.137-158.

WARHURST, Chris; NICKSON, Dennis. Who's Got the Look? Emotional, Aesthetic and Sexualized Labour in Interactive Services. Gender, Work and Organization, vol. XVI, n. 3, 2009. 